\title{
A sensor measuring deformation and pressure, entirely biodegradable, for orthopedic applications
}

\author{
Clementine M. Boutry \\ Bob C. Schroeder \\ Zhenan Bao \\ Department of Chemical Engineering \\ Stanford University, \\ Stanford, CA, USA
}

\author{
Anaïs Legrand \\ Paige Fox \\ Plastic \& Reconstructive Surgery \\ Stanford University Medical Center, \\ Veteran Hospital, \\ Palo Alto, CA, USA
}

\begin{abstract}
In this paper, we present a highly sensitive sensor, entirely made of biodegradable materials, that can measure both deformation and pressure. This sensor is designed to monitor the progress of tendon repair after surgery, with the objective to provide an appropriate care to each patient based on personalized diagnosis. This is the first time that a biodegradable pressure and strain sensor is presented. Moreover, our device's high sensitivity in the low strain regime and low pressure regime in combination with its fast response time in the millisecond range, and its negligible hysteresis is unprecedented when compared to recent reports on implantable strain and pressure sensors. The pressure sensor performs stably after thousands of cycles. These characteristics allow our pressure sensor to be useful for orthopedics applications.
\end{abstract}

Keywords-strain sensor, pressure sensor, tendon repair monitoring, tendon surgery, biodegradable sensor, resorbable sensor, implant

\section{INTRODUCTION}

A new class of sensors, designed for being implanted inside the human body and completely made of biodegradable materials, is currently emerging. These sensors open the field for new diagnostic techniques, without need for a second surgery to remove the devices after their predefined period of use.

In the present work, we demonstrate a highly sensitive sensor, entirely made of biodegradable materials, that can measure both strain and pressure. This sensor is designed to monitor the progress of tendon repair after surgery. Classical surgical techniques include sewing the torn ends of the tendon and performing an allograft in case of largely damaged tissues. In addition, new treatments are under investigation at Stanford, where an hydrogel loaded with various bio-species is injected locally in order to stimulate the cellular growth. It is of great

C.M.B. acknowledges postdoctoral fellowship support by the Swiss National Science Foundation (postdoc mobility fellowship No. P2EZP2_152118) and the European Commission (Marie-Curie international outgoing fellowship Grant No. 622362). Z.B. acknowledges funding support from the MSIP (Ministry of Science, ICT and Future Planning), Korea, under the "IT Consilience Creative Program" (NIPA-2014-H0201-14-1001) supervised by the NIPA interest for surgeons to monitor in situ the recovery after surgery and the healing status of the tissues, with the objective to provide an appropriate care to each patient based on personalized diagnosis.

The biodegradable sensor, fixed on the tendon, can measure both strain and pressure. The strain is measured with two thin film comb electrodes sliding relative to each other, mounted between two highly flexible elastomer layers. The pressure is measured with a flexible capacitor, where the key element is a microstructured elastic dielectric layer placed between the top and bottom electrode.

Only few examples of biodegradable pressure sensors and strain pressure sensors were published so far. A biodegradable pressure sensor was reported by Luo et al. [1] in which the microfabricated wireless RF pressure sensor consisted of a parallel-plate capacitor filled with air, connected to an inductor coil [1]. Despite its simplicity, this air gap approach suffers from poor sensitivity in the low pressure regime (sensitivity $0.0006 \mathrm{kPa}^{-1}$ in the pressure range $0<\mathrm{p}<20 \mathrm{kPa}$ ) [1]. To improve the sensitivity by several orders of magnitude, we presented a new approach with a biodegradable and biocompatible pressure sensor based on a microstructured dielectric layer [2]. The dielectric consists of a thin, regularly microstructured rubber that is highly compressible, which enables the device to have high pressure sensitivity and fast response time. Sensitivities as high as $0.76 \mathrm{kPa}^{-1}$ were achieved [2]. Regarding strain sensors, a biocompatible strain sensor was demonstrated by Xu et al. [3] with the development of 3D multifunctional membranes for cardiac measurements and stimulation. Strain gauges mounted on stretchable membranes and based on silicon $(\mathrm{Si})$ were presented. If mounted on a biodegradable substrate, these strain sensors could be made resorbable, being used as transient electronics [3]. Recent research shows promising results on the biocompatibility of silicon after degradation inside the body. However, long-term adverse effects of silicon after degradation are still not entirely elucidated $[4,5]$.

In the present work, a sensor combining strain and pressure sensing capabilities, with high sensitivity, low hysteresis, longterm stability, and made of biodegradable materials, is characterized, towards application in orthopedics, more precisely tendon tissues recovery monitoring [2]. 

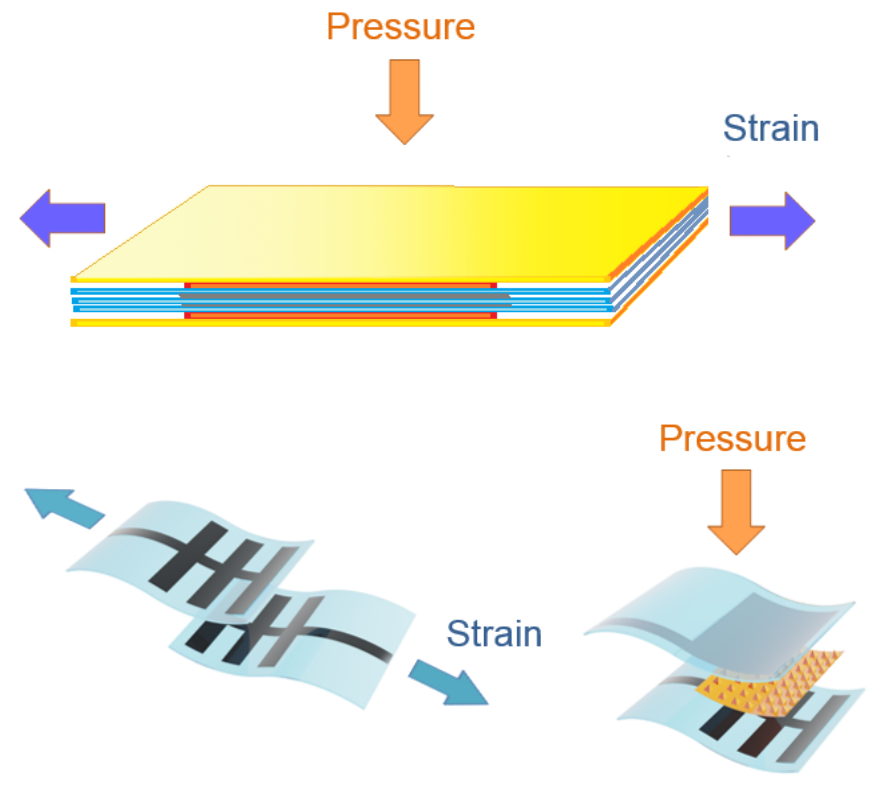

Fig. 1. Concept proposed for the fully biodegradable strain and pressure sensor.

\section{SENSOR CONCEPT AND ASSEMBLY}

\section{A. A fully biodegradable strain and pressure sensor}

Fig. 1 shows the concept proposed for the biodegradable strain and pressure sensor presented in this paper. The biodegradable sensor, fixed on the tendon, can measure both strain and pressure. The strain is measured with two thin film comb electrodes sliding relative to each other, mounted between two highly flexible elastomer layers. The pressure is measured with a flexible capacitor, where the key element is a microstructured elastic dielectric layer placed between the top and bottom electrode.

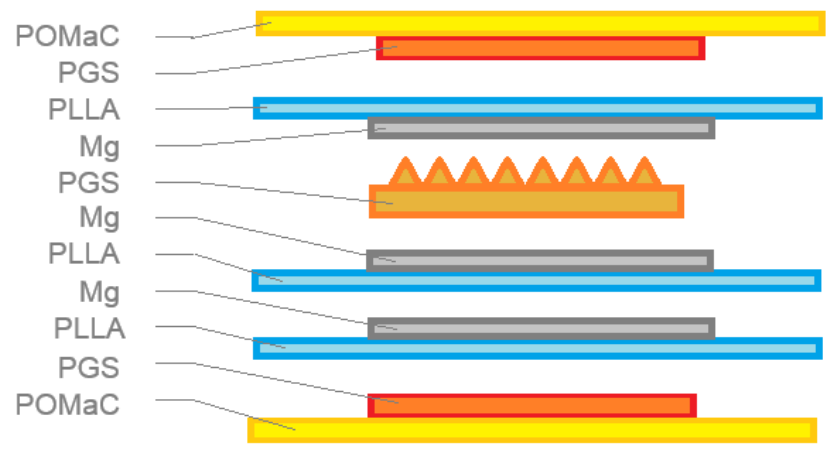

Fig. 3. Material selection for the fully biodegradable strain and pressure sensor.
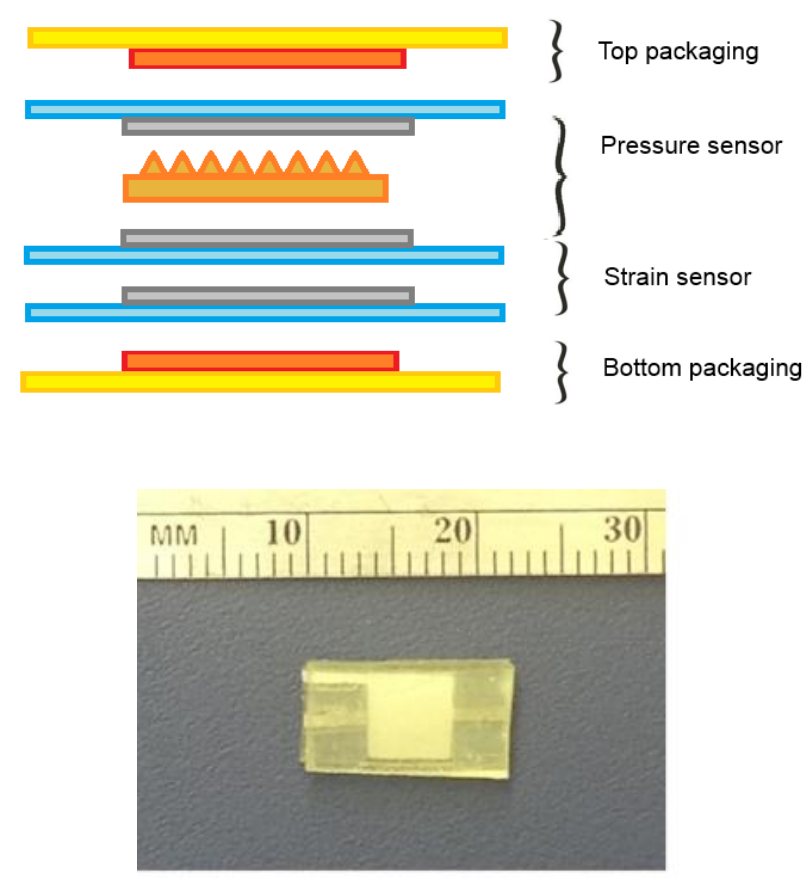

Fig. 2. Overall assembly of the fully biodegradable strain and pressure sensor, and picture of the sensor.

\section{B. Overall assembly and materials selection}

Fig. 2 and Fig. 3 show the overall assembly strategy and the material selection, respectively. The sensor is assembled via lamination process. For the pressure sensor, a key element of the material design is the elastic dielectric between the top and bottom electrodes. Poly(glycerol sebacate) (PGS) is a biodegradable elastomer first reported by Wang et al. for tissue engineering applications [6]. It is an excellent candidate for this application in terms of the degradation characteristics, the mechanical properties, and the processing characteristics. It has been approved by the US Food and Drug Administration (FDA) for biomedical use, which reduces the timeline for implementation. For the strain sensor and packaging, the key element of our material design is $\mathrm{POMaC}$, a biodegradable elastomer [7]. POMaC is a soft biodegradable elastomeric biomaterial synthesized from citric acid, maleic anhydride, and 1,8-octanediol, poly(octamethylene maleate (anhydride) citrate) (POMaC), which is able to mimic the mechanical properties of a wide range of soft biological tissues [7]. The electrodes are made of biodegradable metals evaporated on top of biodegradable polymer substrates. Magnesium is used for the top and bottom electrodes. This biodegradable metal was chosen because of its easy processing, biocompatibility, and rapid rates of hydrolysis. The biodegradable polymer substrate consists of a laser-cut $50 \mu \mathrm{m}$ thick polylactic acid layer (PLLA, Goodfellow, USA). 


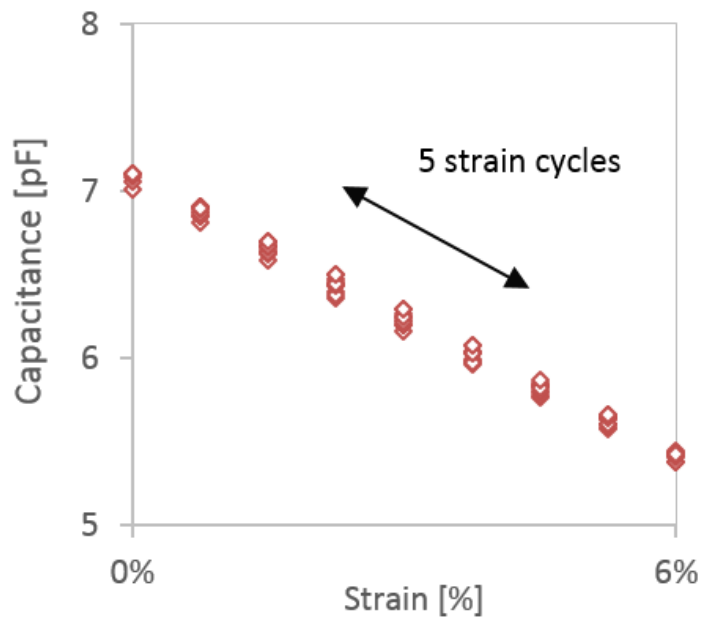

Fig. 4. Strain response curves from 5 consecutive linear loading-unloading cycles.

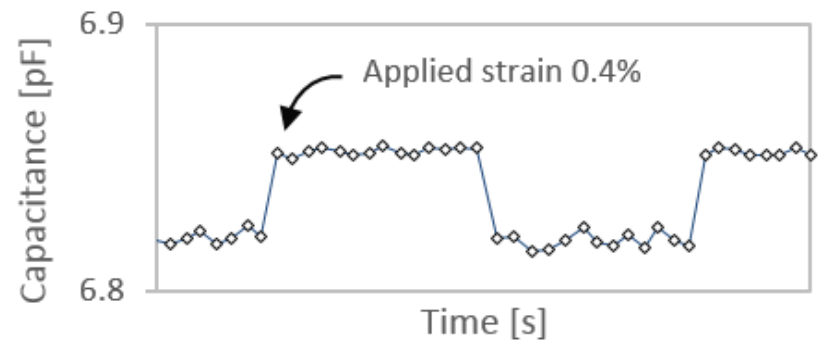

Fig. 5. Smallest detectable strain (no averaging applied, real time measurement).

\section{STRAIN SENSOR CHARACTERIZATION}

Fig. 4 shows the response of the strain sensor to applied strain, for 5 consecutive linear loading-unloading cycles (applied strain 0 to $6 \%$ ). It is observed that the hysteresis is negligible. Fig. 5 shows the smallest detectable strain. Here, a strain of $0.4 \%$ is applied. The SNR is $>2.1$, above the sensor detection limit.

In Fig. 6, the long term response of the strain sensor is evaluated, with applied strain in the range $5 \%$ to $10 \%$. A stable response is measured above $10^{\prime} 000$ cycles. Regarding the achieved sensitivity, for a $15 \%$ strain applied, the relative capacitance change is $\triangle C / C O \sim 50 \%$.

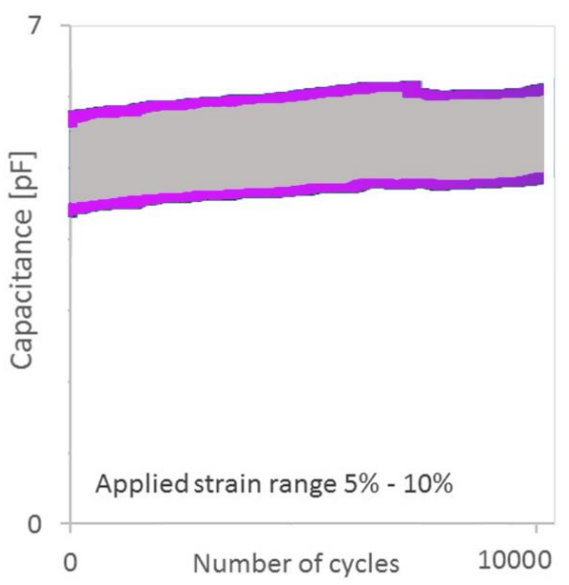

Fig. 6. Long term response of the strain sensor.

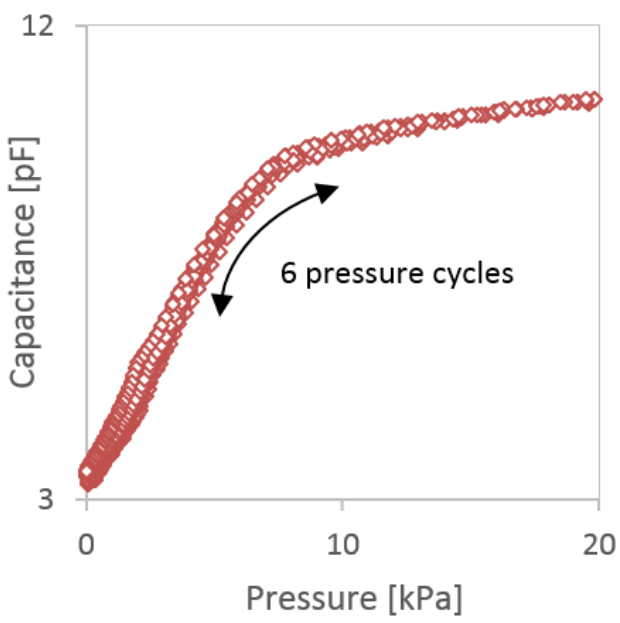

Fig. 7. Pressure response curves from 6 consecutive linear loading-unloading cycles.

\section{PRESSURE SENSOR CHARACTERIZATION}

Fig. 7 shows the response of the pressure sensor to applied pressure, for 6 consecutive linear loading-unloading cycles (applied pressure $20 \mathrm{kPa}$ ). It is observed that the hysteresis is negligible. Fig. 8 shows the smallest detectable pressure. A pressure as small as $12 \mathrm{~Pa}$ is successfully detected by the sensor. In Fig. 9, the long term response of the pressure sensor is evaluated, with applied pressure in the range $15 \mathrm{kPa}$ to 45 $\mathrm{kPa}$. A stable response is measured above 1'000 cycles. The sensitivity is measured to be $0.7 \mathrm{kPa}^{-1}$ in the pressure range 0.1 to $1 \mathrm{kPa}, 0.3 \mathrm{kPa}^{-1}$ in the range $2-5 \mathrm{kPa}$, and $0.03 \mathrm{kPa}^{-1}$ in the pressure range $15-18 \mathrm{kPa}$, very similar to previously published performances for non-degradable versions of the pressure sensor [2]. 


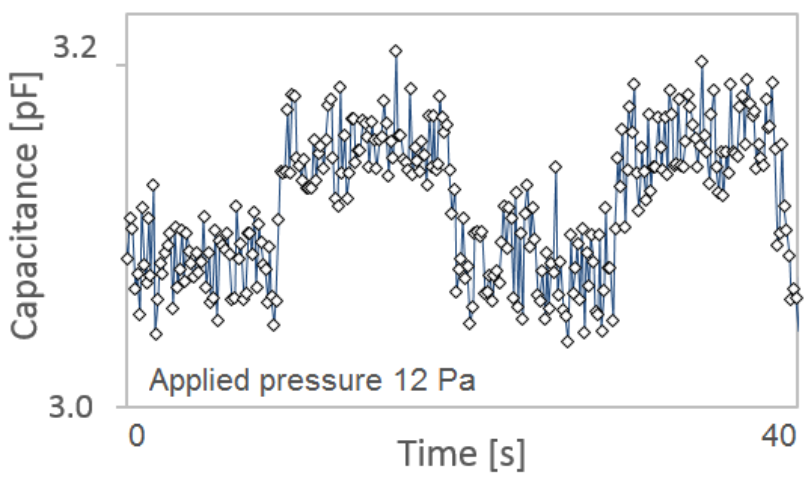

Fig. 8. Smallest detectable pressure (no averaging applied, real time measurement).

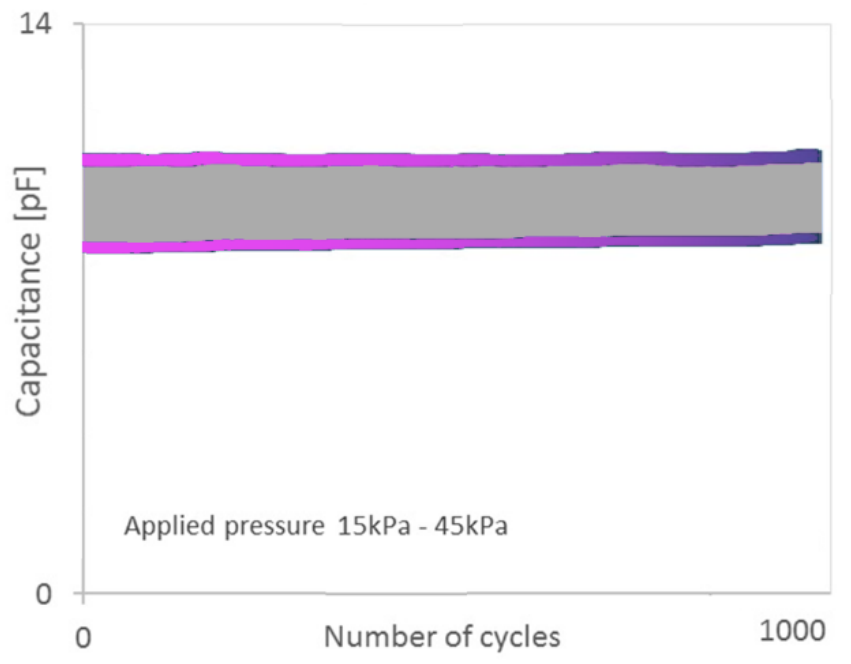

Fig. 9. Long term response of the pressure sensor.

\section{CONCLUSION}

We have presented a strain and pressure sensor, entirely made of biodegradable materials. Our device's high sensitivity in the low strain regime (strains as low as $0.4 \%$ are detected with sensitivities of typically $\Delta \mathrm{C} / \mathrm{C} 0 \sim 50 \%$ for a $15 \%$ strain applied) and low pressure regime $(0.7 \mathrm{kPa}-1$ in the range 0.1 to $1 \mathrm{kPa}, 0.3 \mathrm{kPa}-1$ in the range $2-5 \mathrm{kPa}$, and $0.03 \mathrm{kPa}-1$ in the range $15-18 \mathrm{kPa}$ ), in combination with its fast response time in the millisecond range, and its negligible hysteresis is unprecedented when compared to recent reports on implantable strain and pressure sensors. The pressure sensor performs stably after thousands of cycles. These characteristics allow our pressure sensor to be useful for orthopedics applications.

\section{ACKNOWLEDGMENT}

Part of this work was performed at the Stanford Nano Shared Facilities (SNSF).

\section{REFERENCES}

[1] M. Luo , A. W. Martinez , C. Song , F. Herrault , M. G. Allen , and J. Microelectromech., "A Microfabricated Wireless RF Pressure Sensor Made Completely of Biodegradable Materials", Syst., vol. 23 , pp. 4, 2014.

[2] C. M. Boutry, A. Nguyen, Q. Omotayo Lawal, A. Chortos, S. RondeauGagné, Z. Bao, "A sensitive and biodegradable pressure sensor array for cardiovascular monitoring," Advanced Materials, available online, 2015.

[3] L. Xu , S. R. Gutbrod, A. P. Bonifas, Y. Su, M. S. Sulkin, N. Lu, H. J. Chung, K. I. Jang, Z. Liu , M. Ying, C. Lu , R. C. Webb , J. S. Kim , J. I. Laughner, H. Cheng, Y. Liu , A. Ameen, J. W. Jeong, G. T. Kim , Y. Huang, I. R. Efi mov, J. A. Rogers, "3D multifunctional integumentary membrane for spatiotemporal cardiac measurements and stimulation across the entire epicardium", Nat. Commun., vol. 25 , pp. 3329, 2014.

[4] S. W. Hwang, H. Tao , D. H. Kim , H. Cheng, J. K. Song, E. Rill , M. A. Brenckle , B. Panilaitis , S. M. Won , Y. S. Kim , Y. M. Song, K. J. Yu , A. Ameen , R. Li , Y. Su , M. Yang, D. L. Kaplan , M. R. Zakin , M. J. Slepian, Y. Huang, F. G. Omenetto , J. A. Rogers , "A Physically Transient Form of Silicon Electronics" Science, vol. 28 , pp. 1640, 2012.

[5] S.-K. Kang, G. Park, K. Kim, S.-W. Hwang, H. Cheng, J. Shin, S. Chung, M. Kim, L. Yin, J. Chul Lee, K.-M. Lee, and J. A. Rogers, "Dissolution Chemistry and Biocompatibility of Silicon- and Germanium-Based Semiconductors for Transient Electronics", ACS Appl. Mater. Interfaces, vol. 7 (17), pp. 9297-9305, 2015.

[6] Y. Wang , G. A. Ameer , B. J. Sheppard , R. Langer , "A tough biodegradable elastomer" Nat. Biotechnol., vol. 20 , pp. 602, 2002.

[7] R. T. Tran, P. Thevenot, D. Gyawali, J.-C. Chiao, L. Tanga and J. Yang, "Synthesis and characterization of a biodegradable elastomer featuring a dual crosslinking mechanism", Soft Matter, vol. 6, pp. 2449-2461, 2010 . 